Meta

Journal des traducteurs

Translators' Journal

\title{
SUSAM-SARAEVA, Şebnem (2015): Translation, popular music and transcultural intimacy in Turkish-Greek relations. Oxford: Peter Lang, 180 p.
}

\section{Rocío García Jiménez}

Volume 62, numéro 2, août 2017

URI : https://id.erudit.org/iderudit/1041038ar

DOI : https://doi.org/10.7202/1041038ar

Aller au sommaire du numéro

\section{Éditeur(s)}

Les Presses de l'Université de Montréal

ISSN

0026-0452 (imprimé)

1492-1421 (numérique)

Découvrir la revue

Citer ce compte rendu

García Jiménez, R. (2017). Compte rendu de [SUSAM-SARAEVA, Sebnem (2015):

Translation, popular music and transcultural intimacy in Turkish-Greek

relations. Oxford: Peter Lang, 180 p.] Meta, 62(2), 474-475.

https://doi.org/10.7202/1041038ar d'utilisation que vous pouvez consulter en ligne.

https://apropos.erudit.org/fr/usagers/politique-dutilisation/ 
bienséance de son époque et la condamnation de la traduction littérale comme étant une superstition, s'ajoute une troisième [...], le statut de la matière traduite» (p. 94). Dans les conclusions sur l'analyse paratextuelle, nous apprenons qu'Herberay se "délivre peu à peu des liens de la "fidélité" » au moyen de «la stratégie discursive et éditoriale mise en place dans les textes liminaires du premier livre de l'Amadis» (p. 149).

Enfin, pour s'assurer d'aller au-delà de l'éternel débat de la fidélité en traduction, García Barrera se sert des fondements théoriques qu'il a exposés dans les deux premières parties et marie notamment les propos de Berman sur l'horizon traductif et de Toury sur les normes et les périphéries. Il tisse ainsi des liens entre le texte, le contexte et le sujet traduisant. Pour García Barrera, le contexte est lié au texte «à travers la subjectivité du traducteur. [...] Par conséquent, contextualiser une traduction ne consiste pas tout à fait à étudier son contexte, mais surtout la manière dont le sujet traduisant perçoit et intègre cet environnement dans sa réécriture» (p. 86). Ces propos servent de lignes directrices dans son analyse de la traduction d'Herberay qu'il ne cherche pas à critiquer, mais plutôt à comprendre. En effet, il reprend en partie la terminologie des "tendances déformantes» de Berman pour réaliser une confrontation textuelle linéaire. Même si son analyse ne se veut pas une appréciation de la qualité de la traduction, l'auteur reconnaît le caractère subjectif de sa démarche. «Bien que notre visée est descriptive plutôt que critique, l'observation des différences entre les deux textes qui nous concernent n'a pas moins un caractère subjectif et variable qu'il ne conviendrait pas d'ignorer» (p. 170).

Si Herberay décrit sa traduction de l'Amadis comme une lecture d'agrément, le volume de García Barrera est plutôt une lecture-investissement en raison de la rigueur scientifique du travail. À elle seule, la démonstration des fondements théoriques saurait préparer les chercheurs de tout âge à l'analyse d'un corpus de textes anciens. Un regret pourtant: nous aurions apprécié un résumé des notions théoriques sur la mise en page des textes imprimés de la Renaissance, aspect essentiel de l'analyse textuelle d'œuvres de cette période. En conclusion, García Barrera répond enfin à la question initiale de manière complète:

«Traduire» l'Amadis de Gaule en 1540 voulait dire reprendre l'intention qui animait et qui configurait le texte de départ, perfectionner la mise en cuvre de cette intention par l'imitation, et ce faisant, poursuivre la translation des Amadis et, comme Montalvo un demisiècle avant, les faire "renaître" pour un lecteur nouveau. (p. 296)
C'est ainsi que l'auteur met fin à sa réflexion profonde sur le statut de la traduction et du sujet traduisant en France en 1540.

Marie-France Guénette Université de Montréal, Montréal, Canada

\section{RÉFÉRENCES}

Bideaux, Michel (1998): Vérité et fiction dans les liminaires des Amadis de Gaule (I. I-VIII) Razo. Cahiers du centre médiéval de Nice. 15:93-103.

Guillerm, Luce (1988): Sujet de l'écriture et traduction autour de 1540. Paris: Aux Amateurs de livres, $609 \mathrm{p}$.

Simonin, Michel (1984): La disgrâce d'Amadis. Studi frances.i 28(82):1-35.

Susam-Saraeva, Şebnem (2015): Translation, popular music and transcultural intimacy in Turkish-Greek relations. Oxford: Peter Lang, $180 \mathrm{p}$.

La Dra. Susam-Saraeva, de la Universidad de Edimburgo, es en la actualidad una de las investigadoras que más ha reflexionado sobre la relación entre música y traducción. Prueba de ello es el volumen que la revista The Translator dedicó en 2008 al tema y de cuya coordinación y edición se encargó dicha investigadora.

Además, Susam-Saraeva es una de las pocas autoras (junto con otros, como Franzon -2001, 2005-, Kaindl -2004, 2005- o Low -2003, 2005, 2017-, por citar algunos) que más atención ha prestado a la traducción de canciones de música pop, algo que no ha despertado un especial interés dentro de la traducción de productos musicales, si se compara con otras modalidades, como la traducción de ópera, por ejemplo, la cual goza de una mayor popularidad dentro de los círculos académicos traductológicos.

En el presente libro, Susam-Saraeva sigue ahondando, por una parte, en la relación entre música, traducción y sociedad y, por otra, en los rasgos que delimitan y definen la traducción de canciones pop. A su vez, la autora se propone conseguir que dichos rasgos se establezcan como bases para la práctica y el estudio de esta modalidad de traducción, tanto de manera específica como general. Para llevar a cabo este objetivo, la obra parte de un estudio de caso centrado en la traducción de canciones turcas y griegas. De este modo, a través de un análisis tan concreto, a simple vista, como puede ser el de la traducción de canciones pop a ambos lados del Egeo, se construirá (con gran maestría) un marco general en el que tendrá cabida cualquier aspecto de la traducción de música pop. 
Una de las claves de este trabajo tiene que ver con el concepto de "intimidad transcultural" ("transcultural intimacy”, empleado, según indica la propia Susam-Sareva, por Bigenho 2012, Herzfeld 1997, o Stokes 2010). Gracias a este, la autora será de capaz de trasmitir a un público académico internacional nociones intrínsecas a la cultura de masas propias de un país (o de dos, en este caso), solo comprendidas, en principio y de forma nostálgica y sentimental, por griegos y turcos.

La elección del estudio de caso está íntimamente ligada, tal y como se explica al lector al inicio de la obra, no solo a motivos de carácter biográfico, sino también a motivos sociológicos, ya que la música griega ha tenido gran importancia en la sociedad turca a finales del siglo XX y principios del XXI. Susam-Saraeva creció escuchando canciones griegas y turcas y posee un vasto conocimiento sobre la relación musical entre ambos países, el cual queda plasmado en las numerosas y continuas referencias discográficas y bibliográficas. Debido a esta amplia cultura, no solo traductológica, sino también musical y sociológica, el análisis realizado en el libro se va a extender y entrelazar con otras muchas disciplinas, como son el estudio de la música popular, la etnomusicología, los estudios griegos y turcos, la sociología de la música o el tan actual y novedoso fenómeno fan. Esta inter y multidisciplinariedad queda patente en la manera en que está estructurada la obra, donde además se mezclan el estudio de las diferentes etapas que afectan a la producción y consumo de música pop con las diferentes técnicas o estrategias de traducción que se pueden dar este género musical.

Uno de los capítulos más innovadores del libro es el que trata el fenómeno fan, aplicado en este caso a los foros de traducción de canciones pop en Internet. La autora examina y describe de forma esclarecedora muchos de los elementos que caracterizan esta práctica social y traductora, como, por ejemplo, el funcionamiento de los foros (uso de alias, imágenes o símbolos por parte de sus integrantes), el ambiente cordial y respetuoso que suele imperar en estos medios de comunicación o el uso del inglés como lingua franca o de enlace cuando surgen dudas de significado.

En sus conclusiones, Susam-Saraeva insiste en la importancia sociopolítica de la música pop y la traducción. Durante toda la investigación, la autora ha hecho especial hincapié en cómo la música y su traducción (ya sea lingüística o no) interfieren en el acercamiento de culturas y en la ruptura de barreras sociales o prejuicios. Esta obra es, sin lugar a dudas, una prueba de ello.

Rocío García JimÉnEZ Universidad de Málaga, Málaga, España

\section{REFERENCIAS}

Franzon, Johan (2001): The Pseudotranslation of Popular Songs, in Pirjo Kukkonen \& Ritva Hartama-Heinonen (eds.) (2001): Mission, Vision, Strategies and Values. A Celebration of Tranlator Training and Translation Studies in Kouvola. Helsinki: Helsinki University Press: 33-44.

Franzon, Johan (2008): Choices in Song Translation. Singability in Print, Subtitles and Song Performance. The Translator 14 (2): 373-99.

KaIndL, Klaus (2004): Die Welt ist schön, Mylord: Zum Genre- und Diskurstranfer in der Popularmusik, in Ina Müller (ed.) (2004): Und sie bewegt sich doch! Heidemarie Salevsky zum 60.Geburstag. Frankfurt am Main: Peter Lang: 177-96.

KaIndL, Klaus (2005): The Plurisemiotics of Pop Song Translation: Words, Music, Voice and Image, in Dinda L. Gorlée (ed.) (2005): Song and Significance, Virtues and Vices of Vocal Translation. Amsterdan / New York: Rodopi: 235-62.

Low, Peter (2003a): Singable Translation of Songs. Perspectives 11(2): 87-103.

Low, Peter (2003b): Translating Poetic Songs. An Attempt to Functional Account of Strategies. Target 15(1): 95-115.

Low, Peter (2005): The Pentathlon Approach to Translating Songs, in Dinda L. Gorlée (ed.) (2005): Song and Significance. Virtues and Vices of Vocal Translation. Amsterdam / New York: Rodopi: 185-212.

Low, Peter (2017): Translating Song. Lyrics and Text. New York: Routledge.

Brzozowski, Jerzy (2015): Autour de la traduction. Paris: Orizons, 280 p.

It's been argued recently by some translation scholars (Chesterman 2006, Simeoni 2007 among others) that the observable expansion of Translation and Interpretation Studies (TIS) over the past few decades was due to a series of paradigm shifts or "turns" which marked the transition of the Western TIS from the narrowly linguistic context towards much broader horizons of "poststructural" multidisciplinarity. However, the recent turns towards cultural, social or gender studies had some visible side effects on the contemporary human sciences. On the one hand, the abovementioned turns in human sciences have eroded the domain boundaries thus leading to the "end of theory" in its traditional understanding. On the other hand, the retreat from the "old school" formalist paradigm has led to significant depreciation of some formalist approaches developed within other scholarly practices and to their consequent elimination from the map of legitimate research 